\title{
QUADRATIC ISOPERIMETRIC INEQUALITY FOR MAPPING TORI OF POLYNOMIALLY GROWING AUTOMORPHISMS OF FREE GROUPS
}

\author{
N. MACURA
}

\begin{abstract}
We prove that the mapping torus $F_{n} \rtimes_{\phi} \mathbb{Z}$ of a polynomially growing automorphism $\phi: F_{n} \rightarrow F_{n}$ of finitely generated free group $F_{n}$ satisfies the quadratic isoperimetric inequality.
\end{abstract}

\section{Introduction}

Isoperimetric functions for finitely presented groups bound the complexity of the word problem by giving a bound on the (minimal) number of relators which one must apply in order to show that a word $w$ in the given generators represents the trivial element in the group. These bounds are given in terms of the length of $w$ (see [Br, Dehn functions and Van Kampen diagrams]). Isoperimetric functions are, up to equivalence, geometric properties of groups, i.e. they are invariant under quasi-isometries. Gromov has shown that a group is word-hyperbolic if and only if it has a linear isoperimetric function. Moreover, it follows from the theorem of Gromov and Ol'shanskii that a group which possesses a subquadratic isoperimetric function is hyperbolic. CAT(0) groups, automatic groups, and $(2 n+1)$ dimensional integral Heisenberg groups, $n \geq 2$, have quadratic isoperimetric functions. There are many examples of groups with exponential isoperimetric functions, among them Baumslag-Solitar groups [ECHLPH].

In this paper we study mapping tori $F_{n} \rtimes_{\phi} \mathbb{Z}$ of polynomially growing (outer) automorphisms $\phi: F_{n} \rightarrow F_{n}$ of finitely generated free groups $F_{n}$ and prove that they satisfy the quadratic isoperimetric inequality.

Let $X$ be a finite cell complex and $\tilde{X}$ its universal cover. Assigning each edge of $X$ a length of one induces a combinatorial metric on the one-skeleton on $\tilde{X}$ which may be extended to metric $d_{\tilde{X}}$ on $\tilde{X}$. There is a similar notion of combinatorial area in $X$. For an edge path $c \subset X$ or $c \subset \tilde{X}$ denote by $|c|$ the combinatorial length of $c$. A function $f: \mathbb{N} \rightarrow \mathbb{N}$ is an isoperimetric function for $X$ if for all $n$ and all closed edge paths $c \subset \tilde{X}$ with $|c| \leq n$ 
there is a disk $\Delta: D^{2} \rightarrow \tilde{X}$ with combinatorial area $\operatorname{Area}(\Delta) \leq f(n)$. We say that $X$ satisfies the quadratic isoperimetric inequality if it possess a quadratic isoperimetric function. A group satisfies quadratic isoperimetric inequality if it is the fundamental group of a finite cell complex which satisfies quadratic isoperimetric inequality.

We prove the following result:

Theorem 1.1. Let $\phi: F_{n} \rightarrow F_{n}$ be a polynomially growing automorphism. Then $F_{n} \rtimes_{\phi} \mathbb{Z}$ satisfies the quadratic isoperimetric inequality.

Since the groups $F_{n} \rtimes_{\phi_{1}} \mathbb{Z}$ and $F_{n} \rtimes_{\phi_{2}} \mathbb{Z}$ are isomorphic if $\phi_{1}: F_{n} \rightarrow F_{n}$ and $\phi_{2}: F_{n} \rightarrow F_{n}$ differ by an inner automorphism we can talk about isoperimetric functions for mapping tori of outer automorphisms of free groups.

The recent development of the theory for $\operatorname{Out}\left(F_{n}\right)$, the group of outer automorphisms of a free group on $n$ generators, is modeled on the NielsenThurston theory for surface homeomorphisms. While the well-studied mapping classes have only exponential or linear growth, outer automorphisms of free groups can have exponential or polynomial growth where the degree of the polynomial can be any integer between 1 and $n-1$ for a free group on $n$ generators (see $[\mathrm{BH}]$ ). An outer automorphism $\mathcal{O} \in \operatorname{Out}\left(F_{n}\right)$ is polynomially growing if for each conjugacy class $[\gamma]$ in $F_{n}$ the sequence of (cyclically reduced) word lengths of $\mathcal{O}^{i}([\gamma])$ is bounded above by a polynomial [BFH2].

Recent results of Bestvina, Feighn, and Handel $[\mathrm{BH}],[\mathrm{BFH} 1,2]$ give a nice topological representative for a polynomially growing automorphism of finitely generated free groups $F_{n}$. This enables us to study those groups and answer questions that would otherwise be very difficult to answer. In section 2 we introduce Kolchin maps, maps of finite graphs with special filtrations, where each level in the filtration is built from a previous one by adding a single edge, and such that the map behaves well with respect to that filtration. We require that these maps satisfy a special property which we call the bounded eigenray cancellation property (see Definition 2.6) and which, roughly speaking, means that the possible cancellations between iterates under $f$ of different edges in $G$ are limited. The definition of Kolchin maps is motivated by the above-mentioned results of Bestvina, Feighn and Handel $[\mathrm{BFH} 1,2]$, who prove that there is a topological representative for a unipotent polynomially growing automorphism of free groups (which they call a unipotent representative) that satisfies the above properties of Kolchin maps (see Proposition 2.7). Since for any polynomially growing outer automorphism $\mathcal{O} \in \operatorname{Out}\left(F_{n}\right)$ there is a $p \in \mathbb{N}$ such that $\mathcal{O}^{p}$ is a unipotent 
polynomially growing automorphism and the mapping tori of $\mathcal{O}$ and $\mathcal{O}^{p}$ are quasi-isometric, we can assume that a polynomially growing outer automorphism has a topological representative (up to quasi-isometry) which is a Kolchin map. We define growth of a Kolchin map $f: G \rightarrow G$, which turns out to be equivalent to the growth of the (outer) automorphism that $f$ induces on the fundamental group. Besides introducing Kolchin maps we also discuss the properties of mapping tori of Kolchin maps that will be useful in our proof. In section 3 we describe two different decompositions as a graph of spaces of a mapping torus $M_{f}$ of a Kolchin map $f: G \rightarrow G$. We also introduce level maps and edge fibers, two concepts that will play an important role in the remaining part of the paper. In section 4 we derive some nice properties of edge fibers that are consequences of properties of Kolchin maps and which will be used in proofs of our main results.

In the remaining part of the paper we prove the following theorem:

Theorem 1.2. Let $f: G \rightarrow G$ be a Kolchin map. Then the mapping torus $M_{f}=G \times I /_{(x, 0) \sim(f(x), 1)}$ satisfies the quadratic isoperimetric inequality.

Theorem 1.1 is then a corollary of Theorem 1.2 and Proposition 2.7 of section 2. The main idea in proving Theorem 1.2 will be to use the stratified structure of a Kolchin map $f: G \rightarrow G$ that grows polynomially with degree $d$ to decompose a disk in the mapping torus $M_{f}$ into disks that are contained in the mapping torus of a Kolchin map that grows polynomially with degree $d-1$, and apply induction. However, one immediately faces the problem that when a disk is decomposed into smaller disks, new boundary curves will contain the paths along which we have cut our disk and they, in general, may be quite long. Therefore, the central part of our proof will be to show that such an event cannot happen for well chosen disks $\Delta$. In section 6 we will show that the sum of the lengths of new parts of the boundaries is bounded by a multiple of the length of the original boundary path $c$. The quadratic isoperimetric inequality for mapping tori of linearly growing automorphisms of finitely generated free groups is proved in section 7 , which completes the induction and the proof of the main theorem.

It is proved in $[\mathrm{BF}]$ that $F_{n} \rtimes_{\phi} \mathbb{Z}$ is word hyperbolic (and thus satisfies the linear isoperimetric inequality) when $\phi: F_{n} \rightarrow F_{n}$ is irreducible and non geometric. Since Bestvina, Feighn, and Handel [BH], [BFH1,2] prove that for every outer automorphism of a finitely generated free group there is a topological representative with a special filtration, similar to those of Kolchin maps, but where each level in the filtration is built from a previous one by adding a single edge or an exponentially growing stratum, 
one expects all $M_{f}$ for $f$ an automorphism of a finitely generated free group to satisfy the quadratic isoperimetric inequality. This is an interesting contrast with the case of $G L(n, Z)$, which is often compared to $\operatorname{Aut}\left(F_{n}\right)$, since mapping tori of most elements of $G L(n, Z)$ do not satisfy the quadratic isoperimetric inequality. More precisely, in $[\mathrm{BrG}]$ Bridson and Gersten relate the Dehn function of a group of the form $G=\mathbb{Z}^{n} \rtimes_{A} \mathbb{Z}$ for $A \in G l_{n}(\mathbb{Z})$ to the growth of $A$ and show that $G$ satisfies the quadratic isoperimetric inequality if and only if $A \in G l_{n}(\mathbb{Z})$ has finite order.

\section{Kolchin Maps}

We will first define a more general class of filtered graphs and their maps which we will call stratified systems. This will enable us to develop necessary terminology that we need in order to define Kolchin maps.

Let $G$ be a graph and $f: G \rightarrow G$ be a map such that $f$ sends vertices to vertices and edges to immersed nontrivial edge paths. In this section (only) we will assume that all of our paths have endpoints in the vertex set. If $\gamma$ is a path, $[\gamma]$ will denote the unique immersed edge path homotopic to $\gamma$ rel endpoints.

A decomposition $\sigma=\sigma_{1} \sigma_{2} \ldots \sigma_{l}$ of a path $\sigma \in G$ is called a $k$-splitting if

$$
\left[f^{k}(\sigma)\right]=\left[f^{k}\left(\sigma_{1}\right)\right]\left[f^{k}\left(\sigma_{2}\right)\right] \ldots\left[f^{k}\left(\sigma_{l}\right)\right],
$$

and is called a splitting if it is a $k$-splitting for all $k \geq 0$. The end points of $\sigma_{i}$ 's are called the splitting set. As a matter of notation, we will follow [BFH1] and use - to separate subpaths if the separation is a splitting.

Definition 2.1. Let $G$ be a finite graph with no valence one vertices and $f: G \rightarrow G$ be a map such that

(1) the map $f$ sends vertices to vertices and edges to immersed nontrivial edge paths;

(2) there is a filtration $\Phi=G_{0} \subseteq G_{1} \cdots \subseteq G_{k}=G$ of $G$ by $f$ invariant subgraphs such that each $\left(G_{i}\right)$ is the union of $G_{i-1}$ and a single edge $E_{i}$ satisfying $f\left(E_{i}\right)=E_{i} \cdot u_{i}$ for some closed path $u_{i}$ that crosses only edges in $G_{i-1}$.

Then $f: G \rightarrow G$ is called a stratified system. The edge $E_{i}$ is called the $i^{\text {th }}$ stratum. The based loops $u_{i}$ are suffixes of $f$.

Definition 2.2 [BFH2, see Definition 3.14]. Let $f: G \rightarrow G$ be a stratified system and $\tilde{G}$ the universal cover of $G$. The height of an edge-path $\gamma$ in $G$, $\operatorname{height}(\gamma)$, is the smallest $m$ such that the path is contained in $G_{m}$. A 
topmost edge in an edge path of height $m$ is an occurrence of $E_{m}$ or $E_{m}^{-1}$ in the edge path.

If $\gamma$ is a path in the universal cover $\tilde{G}$ of $G$ with the covering map $\pi: \tilde{G} \rightarrow G$ we will say that height $(\gamma)$ is the height of $\pi(\gamma)$. A topmost edge in an edge path $\gamma \in \tilde{G}$ of height $m$ is an occurrence of $\tilde{E}_{m}$ or $\tilde{E}_{m}^{-1}$ in the edge path. We will use the terminology introduced in [BFH1,2] for $U R-$ topmost edges, height, etc., whenever it also applies for stratified systems.

We will need the following lemma, which is proved in a more general form in [BFH1, Lemma 4.1.4].

Lemma 2.3 (see [BFH1, Lemma 4.1.4]). Suppose that $f: G \rightarrow G$ is a stratified system and that $f\left(E_{i}\right)=E_{i} u_{i}$ for some closed path $u_{i} \subset G_{i-1}$. Suppose also that $\sigma \subset G_{i}$ is an edge path or circuit that intersects $G_{i}$ nontrivially. Then $\sigma$ can be split into subpaths of the following type, where $\gamma$ is a subpath in $G_{i-1}: \gamma, E_{i} \gamma E_{i}^{-1}, E_{i} \gamma$, or $\gamma E_{i}^{-1}$.

Following [BFH1] we will refer to these four types of paths as basic paths.

Definition 2.4 [BFH2, Definition 3.11]. Let $f: G \rightarrow G$ be a stratified system. A path $\tau$ in $G$ with endpoints in the vertex set is Nielsen if $[f(\tau)]=[\tau]$.

Let $f: G \rightarrow G$ be a stratified system. Since each suffix $u_{i}$ is a loop, iterated application of $f$ on $E_{i}$ will give us an infinite ray $R_{i}$ which we call the eigenray associated to $E_{i}$. Lifts of $R_{i}$ to the universal cover of $G$ are also called eigenrays. Moreover, if a stratified system $f: G \rightarrow G$ is a topological representative for a unipotent polynomially growing automorphism of a free group, we will be able to control how many iterates of $\tilde{u}_{i}$ in such an eigenray associated to $\tilde{E}_{i}$ can also be contained in an eigenray corresponding to a different edge $\tilde{E}_{m}$. This property will be very important in proving our main results on mapping tori of polynomially growing automorphisms of free groups.

Definition 2.5 [BFH2]. Let $f: G \rightarrow G$ be a stratified system. The immersed infinite ray

$$
R_{i}=E_{i} u_{i}\left[f\left(u_{i}\right)\right] \cdots\left[f^{k-1}\left(u_{i}\right)\right] \cdots
$$

is the eigenray associated to $E_{i}$. Lifts of $R_{i}$ to the universal cover of $G$ are also called eigenrays. The subpaths $\left[f^{k}\left(u_{i}\right)\right]$ are called blocks.

Definition 2.6. A Kolchin map is a stratified system $f: G \rightarrow G$ which satisfies the following bounded eigenray cancellation property: 
Let $P \in \tilde{G}$ and $Q \in \tilde{G}$ be two points contained in the eigenrays corresponding to two different edges $\tilde{E}_{m}, f\left(E_{m}\right)=E_{m} u_{m}$ and $\tilde{E}_{i}, f\left(E_{i}\right)=E_{i} u_{i}$. If the geodesic connecting $P$ and $Q$ contains more than three blocks of the eigenray corresponding to $\tilde{E}_{m}$ and more than three blocks of the eigenray corresponding to $\tilde{E}_{i}$ then $u_{i}=[\tau]^{p}, u_{m}=[\tau]^{q}$ for a Nielsen path $\tau$ and $p, q \in \mathbb{N}$.

Moreover, we require that $f: G \rightarrow G$ is identity if the induced map on the fundamental group is identity.

We will show that for any polynomially growing outer automorphism $\mathcal{O} \in \operatorname{Out}\left(F_{n}\right)$ there is a Kolchin map $f: G \rightarrow G$ such that the mapping torus of $\mathcal{O}$ is quasi-isometric to the universal cover of the mapping torus of $f$. An outer automorphism is a unipotent polynomially growing automorphism if it is a polynomially growing outer automorphism $P G\left(F_{n}\right)$ and its action on $H_{1}(F, \mathbb{Z})$ is unipotent. Since for any polynomially growing outer automorphism $\mathcal{O} \in \operatorname{Out}\left(F_{n}\right)$ there is $p \in \mathbb{N}$ such that $\mathcal{O}^{p}$ is a unipotent polynomially growing automorphism [BFH1], we can restrict our attention to the unipotent polynomially growing automorphisms.

Proposition 2.7 (see $[\mathrm{BFH} 1,2]$ ). Let $\phi: F_{n} \rightarrow F_{n}$ be a unipotent polynomially growing automorphism of a free group $F_{n}$. There is a filtered graph $G$ such that $\phi$ has a representative that is a Kolchin map on $G$.

REMARK 2.8. Theorem 3.12 in [BFH2] states that for any unipotent polynomially growing outer automorphism there is a topological representative that is a stratified system. Sublemma 5.5.2 [BFH1] implies that there is a topological representative for a unipotent polynomially growing outer automorphism that is a stratified system with the bounded eigenray cancellation property.

In the remaining part of this section we discuss the growth of a Kolchin map and give examples.

Definition 2.9. A Kolchin map $f: G \rightarrow G$ grows polynomially with degree $d$ if

- for each path $\gamma \subset G$ there is a polynomial $p_{\gamma}$ of degree $d$ such that the sequence of path lengths $\left|f^{i}(\gamma)\right|$ is bounded above by $p_{\gamma}(i)$;

- there is a path $\omega \subset G$ and a polynomial $q_{\omega}$ of degree $d$ such that sequence of path lengths $\left|f^{i}(\omega)\right|$ is bounded below by $q_{\omega}(i)$.

Note that a Kolchin map that grows polynomially with degree $d \geq 1$ induces an outer automorphism on the fundamental group that grows poly- 
nomially with degree $d$ and therefore the above definition is consistent with Definition 2.9 .

REMark 2.10. Definition 2.9 is equivalent to the following:

- for each edge $c \subset G$ there is a polynomial $p_{c}$ of degree $d$ such that the sequence of path lengths $\left|f^{i}(c)\right|$ is bounded above by $p_{c}(i)$;

- there is an edge $e \subset G$ and a polynomial $q_{e}$ of degree $d$ such that sequence of path lengths $\left|f^{i}(e)\right|$ is bounded below by $q_{e}(i)$.

The above discussion motivates the following definition.

Definition 2.11. Let $f: G \rightarrow G$ be a Kolchin map. An edge $c \subset G$ grows polynomially with degree $d$ if there are polynomials $p_{l}$ and $p_{u}$ of degree $d$ such that

$$
p_{l}(i) \leq\left|f^{i}(c)\right| \leq p_{u}(i), \quad i \in \mathbb{N} .
$$

REMARK 2.12. A Kolchin map $f: G \rightarrow G$ grows polynomially with degree $d$ if all the edges in $G$ grow polynomially with degree less than or equal to $d$ and there is an edge $e \subset G$ that grows polynomially with degree $d$.

We will give some examples of Kolchin maps.

EXAMPLE 2.13. Let $G_{1}$ be a bouquet of two circles and let $f_{1}: G_{1} \rightarrow G_{1}$ be a map that fixes the vertex and sends the edges, labeled $a$ and $b$, onto edge paths as follows:

$$
\begin{aligned}
& f_{1}(a)=a, \\
& f_{1}(b)=b a .
\end{aligned}
$$

It is not difficult to see that $b$ grows linearly and, since $a$ is an invariant under $f_{1}$, we can conclude that $f_{1}$ is a Kolchin map that grows linearly.

ExAmPLE 2.14. Let $G_{2}$ be a bouquet of three circles labeled $a, b$, and $c$, and let $f_{2}: G_{2} \rightarrow G_{2}$ be a map that fixes the vertex and sends the edges onto edge paths as follows:

$$
\begin{aligned}
& f_{2}(a)=a, \\
& f_{2}(b)=b a, \\
& f_{2}(c)=c a a .
\end{aligned}
$$

Then $f_{2}$ is a Kolchin map that grows linearly.

EXAMPLE 2.15. Let $G_{3}$ be a bouquet of three circles labeled $a, b$ and $c$ and let $f_{3}: G_{3} \rightarrow G_{3}$ be a map that fixes the vertex and sends the edges onto edge paths as follows:

$$
\begin{aligned}
& f_{3}(a)=a \\
& f_{3}(b)=b a \\
& f_{3}(c)=c b .
\end{aligned}
$$


In this example $b$ again grows linearly, but $c$ grows quadratically, so $f_{3}$ is a Kolchin map that grows quadratically.

In order to prove our main results we will use induction on the growth of Kolchin maps. For that purpose we need to show that for an edge $E_{n} \subset G$, $f\left(E_{n}\right)=E_{n} \cdot u_{n}$ that grows polynomially with degree $d$ all the edges in $u_{n}$ grow polynomially with degree less than or equal to $d-1$, and that there is an edge $E_{k}$ in $u_{n}$ that grows polynomially with degree $d-1$. We will also show that the filtration on $G$ can be chosen so that it is consistent with the growth of the edges. In other words, the edges that grow with higher degree are contained in higher subgraphs in the filtration. This will imply that the topmost edge in $u_{n}$ grows polynomially with degree $d-1$. In order to do this we will need the following lemma.

Lemma 2.16. Let $E_{n} \subset G$ be an edge such that $f\left(E_{n}\right)=E_{n} \cdot u_{n}$ and assume that $E_{n}$ grows polynomially with degree $d \geq 2$. Then the following holds:

(i) every edge $E_{i}$ in $u_{n}$ grows polynomially with degree less than or equal to $d-1$;

(ii) there is an edge $E_{k}$ in $u_{n}$ that grows polynomially with degree $d-1$.

Moreover, if $d=2$ there is a subpath $\sigma$ of $u_{n}$ which has endpoints in the split set of $u_{n}$ and which grows linearly.

Proof. We will prove claim $(i)$ inductively. Assume that the claim is true for every edge $E_{i}$ with $i=h e i g h t\left(E_{i}\right)<n$. Let $E_{k}$ be the topmost edge in $u_{n}$ and assume that $E_{k}$ grows polynomially with degree $p \geq 2$. As a consequence of the inductive hypothesis, we can assume, after possibly rearranging the filtration, that any other edge in $u_{n}$ grows polynomially with degree less than or equal to $p$. We will prove that $E_{n}$ grows polynomially with degree greater than or equal to $p+1$.

In order to prove our claim, we will show that there is a constant $N \in \mathbb{N}$ such that for any $j \geq N$ the path $\left[f^{j}\left(u_{n}\right)\right]$ contains at least $j-N$ blocks of the eigenray associated with $E_{k}$. This implies that if $E_{k}$ grows polynomially with degree $p$, then $E_{n}$ grows polynomially with degree greater or equal to $p+1$.

Let $E_{k}$ be the topmost edge in $u_{n}$. Lemma 2.3 implies that $u_{n}$ can be split into basic paths, that is subpaths of the following type: $\gamma, E_{k} \gamma \bar{E}_{k}$, $E_{k} \gamma$, or $\gamma \bar{E}_{k}$, for $\gamma$ a subpath in $G_{k-1}$ and $\bar{E}_{k}=E_{k}^{-1}$. We will prove our claim for the basic path of the form $E_{k} \gamma$ since the other cases follow by a similar argument. 
If height $(\gamma) \leq$ height $\left(u_{k}\right)$, we are done since in this case at most $|\gamma|$ blocks of the eigenray associated with $E_{k}$ can be canceled with $f(\gamma)$. (The number of blocks that can be canceled is bounded by the number of times that $\gamma$ crosses $E_{k}$.) Therefore we will assume that height $(\gamma)>\operatorname{height}\left(u_{k}\right)$ and prove our claim by induction on height $(\gamma)$. Let $E_{j}$ be the topmost edge in $\gamma$. Then $E_{k} \gamma$ can be split into subpaths of the following type: $\omega, E_{k} \omega \bar{E}_{j}$, $E_{k} \omega$, or $\omega \bar{E}_{j}$, for $\omega$ a subpath in $G_{j-1}$. We are interested in subpaths that contain $E_{k}$, that is $E_{k} \omega$ or $E_{k} \omega \bar{E}_{j}$. If we have a subpath of the form $E_{k} \omega$, for $\omega$ a subpath in $G_{j-1}$, we can apply induction and the claim follows. In the case $E_{k} \omega \bar{E}_{j}$ we repeat the above procedure, that is consider cases when height $(\omega) \leq \operatorname{height}\left(u_{k}\right)$ or height $(\omega)>$ height $\left(u_{k}\right)$. Similarly as before, in the first case it follows that at most $|\omega|+3$ blocks of the eigenray associated with $E_{k}$ can be canceled with iterates under $f$ of $\omega \bar{E}_{j}$. In the case height $(\omega)>$ height $\left(u_{k}\right)$ we have a splitting at the topmost edge in $\omega$ and the claim follows by induction.

It is not difficult to see that if all the edges (or subpaths with endpoints in the split set of $u_{n}$ ) grew polynomially with degree strictly less than $d-1$, than $E_{n}$ would grow polynomially with degree strictly less than $d$, which proves the claim (ii).

In order to simplify some of our arguments we will require our filtrations to be efficient, which is just a technical requirement on indexing.

Definition 2.17. Let $f: G \rightarrow G$ be a stratified system with a filtration $\Phi=G_{0} \subseteq G_{1} \cdots \subseteq G_{n}=G$ of $G$ by $f$ invariant subgraphs. We say that $\Phi$ is an efficient filtration if whenever $i \leq j$ and $E_{j}$ grows polynomially with degree $d \geq 2$, then $E_{i}$ grows polynomially with degree less than or equal to $d$.

Lemma 2.16 implies that we can assume that a Kolchin map has an efficient filtration. Since the edges that grow with degrees $d$ and $d-1$ will play the most important role in the proofs of our main results, we introduce special terminology for those edges.

Definition 2.18. Let $f: G \rightarrow G$ be a Kolchin map growing polynomially with degree $d$. The collection of topmost edges $\mathcal{E}$ in $G$ is the collection of edges $E_{i} \subset G$ that grow polynomially with degree $d$. An edge $\tilde{E}_{i} \subset \tilde{G}$ belongs to the collection of topmost edges if it is a lift of an edge $E_{i} \subset G$ that grows polynomially with degree $d$. If $E_{j} \subset G$ is the topmost edge in $u_{i}$ for an edge $E_{i} \in \mathcal{E}$ we will say that $E_{j}$ is the dominating edge for $E_{i}$. A dominating edge for an edge $\tilde{E}_{i} \subset \tilde{G}$ is a lift of $E_{j}$ as above.

Note that if $f: G \rightarrow G$ is a Kolchin map growing polynomially with 
degree $d \geq 2$, any dominating edge grows polynomially with degree $d-1$ (2.16). The above statement is not, in general, true if $d=1$. That is, if $E_{i} \subset G$ is a linearly growing edge, the topmost edge $E_{k}$ in $u_{i}$ can also be linearly growing.

\section{The Mapping Torus as a Graph of Spaces}

Throughout the remainder of this chapter we will assume that $f: G \rightarrow G$ is a Kolchin map. We will look at two different decompositions of $M_{f}$ as a graph of spaces: standard decomposition, which comes from the fact that $M_{f}$ is a mapping torus, and the topmost edges decomposition, which comes from the stratified structure of $G$. Standard decomposition will give us important concepts (hyperplanes, edge fibers, and level function) and the topmost edges decomposition will enable us to use induction on the growth of the Kolchin map in proving our results. First we give a definition of graphs of spaces and then we will give descriptions of the two decompositions of $M_{f}$ as a graph of spaces.

Let X be a connected finite cell complex with fundamental group $G$ and let $p: X \rightarrow \Gamma$ be a map onto a finite graph $\Gamma$. Denote the preimage under $p$ of the midpoint of an edge $e$ of $\Gamma$ by $X_{e}$. We require that $X_{e}$ can be bicollared in $X$ with the collaring respecting the projection to the edge $e$. Consider the component containing $v$ of $\Gamma$ cut open along the midpoints of edges. Let $X_{v}$ denote the preimage under $p$ of this component. We further require that each $X_{e}$ and $X_{v}$ be connected and that their inclusions into $X$ induce inclusions of fundamental groups. There is an induced map $\tilde{p}: \tilde{X} \rightarrow T$ from the universal cover of $X$ to a $G$-tree $T$ such that $T / G$ is isomorphic to $\Gamma$. We call $X$ a graph of spaces.

We will first describe the standard decomposition of $M_{f}$ as a graph of spaces, where the graph has one vertex and one edge. The edge space is $G$. The vertex space is obtained from the mapping cylinder $C_{f}=$ $G \times I /(x, 1) \sim f(x)$ by attaching a collar to the (top) $G$.

Let $\tilde{G}$ be the universal covering space of $G$ and let $V(\tilde{G})$ be the set of vertices of $\tilde{G}$. We can identify the 1 -skeleton of $\tilde{C}_{f}$ with

$$
\tilde{G} \times\{0,1\} \cup\left(\cup_{x \in V(\tilde{G})}[x, \tilde{f} x]\right) .
$$

We will label the oriented edges $[x, \tilde{f} x]$ with $\tilde{t}$ and their projections in $M_{f}$ with $t$. Note that the boundaries of 2-cells in $M_{f}$ have labels of the form $t^{-1} E_{i} t f\left(E_{i}\right)^{-1}$ where $E_{i}$ is an edge in $G$.

We will use the standard decomposition of $M_{f}$ as a graph of spaces to 
define level maps $\tilde{g}$ on $\widetilde{M}_{f}$. First note that since $M_{f}=G \times I /{ }_{(x, 0) \sim(f(x), 1)}$, there is a map $g: M_{f} \rightarrow S^{1}$ such that if $[x, t] \in M_{f}$ is the equivalence class of $(x, t)$, then $g([x, t])=t$. This implies that there exists a map $\tilde{g}: \widetilde{M}_{f} \rightarrow \mathbb{R}$ such that $p \tilde{g}=g p_{f}$ where $p_{f}: \widetilde{M}_{f} \rightarrow M_{f}$ and $p: \mathbb{R} \rightarrow S^{1}$ are covering maps. We will call $\tilde{g}$ a level map or level function for $\widetilde{M}_{f}$ and a preimage of a real number $r$ under $\tilde{g}$ an $r$-level in $\widetilde{M}_{f}$. Note that the preimage of a real number $r$ under $\tilde{g}$ is a copy of the universal cover of $G$ so we can talk about eigenrays in any such $r$-level.

We will also define another useful concept, namely edge fibers.

Definition 3.1. Let $B_{m} \subset M_{f}$ be a 2-dimensional cell that has boundary label

$$
t^{-1} E_{m} t f\left(E_{m}\right)^{-1} \text {. }
$$

A spanning arc in $B_{m}$ is a properly embedded arc connecting the midpoint of $E_{m}$ to the midpoint of an edge $e \subset f\left(E_{m}\right)$ (see Figure 1). Let $\tilde{B}_{m} \subset \widetilde{M}_{f}$ be a lift of $B_{m}$. A spanning arc in $\tilde{B}_{m}$ is the lift of a spanning arc in $B_{m}$.

Definition 3.2. An edge fiber $\sigma$ in $\widetilde{M}_{f}$ is an embedded copy of $\mathbb{R}$ such that if $B$ is a 2-cell in $\widetilde{M}_{f}$ and $\sigma \cap B \neq \emptyset$ then $B \cap \sigma$ is a spanning arc or a single point which is a midpoint of an edge $\tilde{E}_{k}$ in the boundary of $B$ (Figure 2).
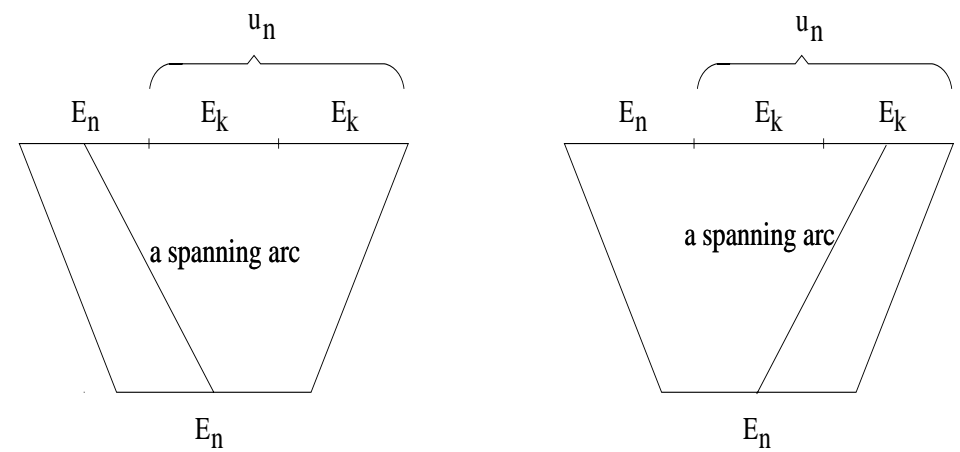

Figure 1: Spanning arcs

When talking about lifts of an edge $E_{i}$ we will allow slight ambiguity, that is sometimes we will use $\tilde{E}_{i}$ to denote a particular edge and sometimes any lift of $E_{i}$. We will make the meaning of the symbol $\tilde{E}_{i}$ clear from the context in any particular situation.

Definition 3.3. An edge fiber $\sigma$ corresponds to an edge $\tilde{E}_{j}$ if 


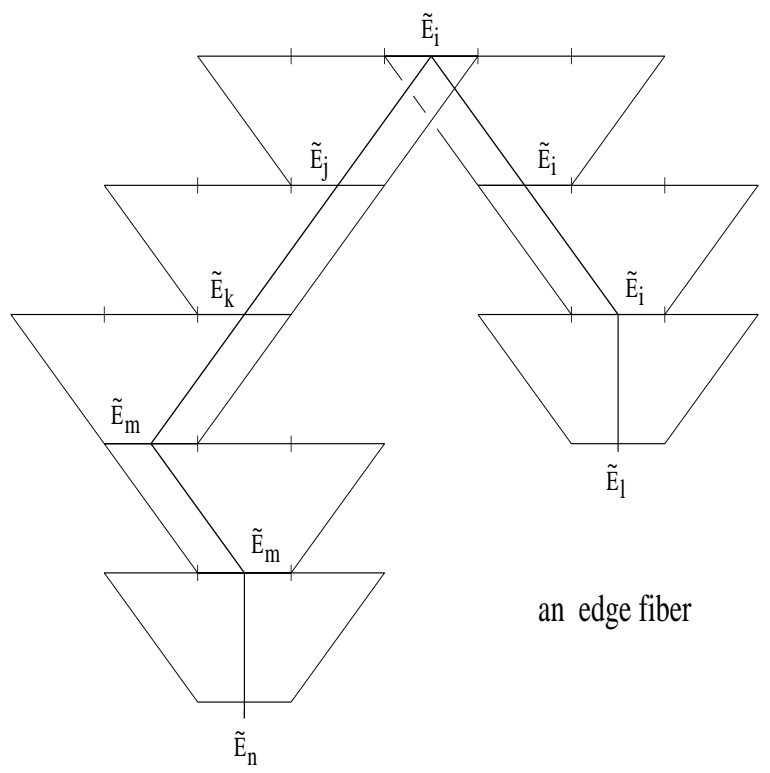

Figure 2: An edge fiber

- $\sigma \cap \tilde{E}_{j} \neq \emptyset$

- $\sigma \cap \tilde{E}_{m} \neq \emptyset$ for $\tilde{E}_{m} \in \tilde{G}$ implies that $m \geq j$.

Definition 3.4. If $\tilde{E}_{m} \subset \tilde{G}$ belongs to the collection of topmost edges then we will call the edge fibers corresponding to $\tilde{E}_{m}$ hyperplanes in $\widetilde{M}_{f}$. If $\tilde{E}_{k} \subset \tilde{G}$ is a dominating edge, we will call the edge fibers corresponding to $\tilde{E}_{k}$ dominating edge fibers.

We can also put a different structure of a graph of spaces on $M_{f}$, where the edges will correspond to the edges in the collection of topmost edges in $G$. Let $f: G \rightarrow G$ be a Kolchin map that grows linearly with degree $d \geq 2$, let $\mathcal{E}=\left\{E_{i}, \ldots, E_{n}\right\}$ be the collection of topmost edges in $G$ and $\mathcal{A}=\left\{a_{i}, \ldots, a_{n}\right\}$ a collection of spanning arcs corresponding to the edges $E_{i}, \ldots, E_{n}$, respectively. Note that every $a_{j} \in \mathcal{A}$ is bicollared in $M_{f}$. Let $A=\cup\left\{a_{i}: a_{i} \in \mathcal{A}\right\}$. We can construct a map $p_{T}: M_{f} \rightarrow \Gamma_{T}$ where $\Gamma_{T}$ is the graph such that the vertices of $\Gamma_{T}$ are connected components of $M_{f} \backslash A$ and edges correspond to the edges in the collection of topmost edges in $G$. This implies that $M_{f}$ has a structure of a graph of spaces where the edge space is the circle $S^{1}$ and vertex spaces are mapping tori of Kolchin maps that grow polynomially with degree strictly less than $d$. We will call this 
decomposition of $M_{f}$ the topmost edges decomposition.

Let $\mathcal{V}$ be the collection of all hyperplanes in $\widetilde{M}_{f}$ and let $V=\cup\left\{s_{j}\right.$ : $\left.s_{j} \in \mathcal{V}\right\}$. Since hyperplanes are the lifts in $\widetilde{M}_{f}$ of spanning arcs in $\mathcal{A}$, that is, the edge spaces in the topmost edges decomposition, the connected components of $\widetilde{M}_{f} \backslash V$ are universal covers of vertex spaces in the topmost edges decomposition of $M_{f}$, and we will call a component $Z$ of $\widetilde{M}_{f} \backslash V a$ vertex component of $\widetilde{M}_{f}$.

Definition 3.5. If $s_{n}$ is a hyperplane let

$$
\operatorname{star}\left(s_{n}\right)=\cup\left\{B: B \text { is a 2-cell in } \widetilde{M}_{f}, B \cap s_{n} \neq \emptyset\right\} .
$$

We can identify the edge $e$ in the graph of spaces with $E_{n}$ and consider it to be an oriented edge. This induces transverse orientation on each hyperplane $s$ and we can talk about two "sides" of $s$, which we will define to be two boundary edge paths of star $(s)$ (Figure 3).

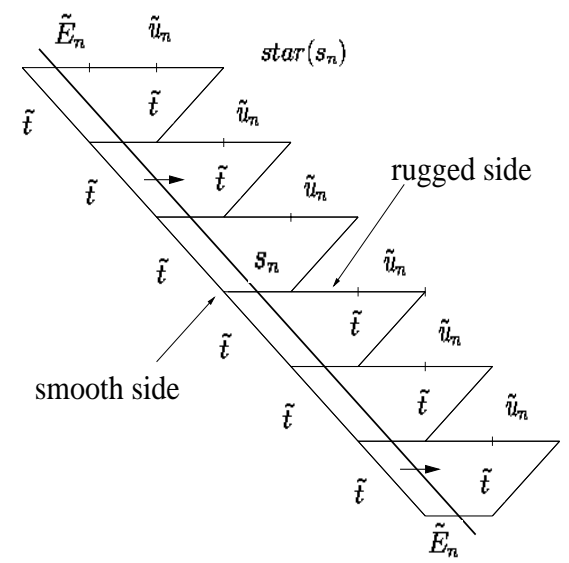

Figure 3: Rugged and smooth sides of a hyperplane $s_{n}$

Note that we have two inclusions of the edge space $S^{1}$ into $M_{f}$, immersions $\phi_{0}, \phi_{1}: S^{1} \rightarrow M_{f}$ that wrap $S^{1}$ once around $t$ and $t u_{n}^{-1}$, respectively. Since the images $\phi_{0}\left(S^{1}\right)=t$ and $\phi_{1}\left(S^{1}\right)=t u_{n}^{-1}$ are reduced edge loops, the induced mappings on the universal covers $\tilde{\phi}_{0}: \mathbb{R} \rightarrow \widetilde{M}_{f}, \tilde{\phi}_{1}: \mathbb{R} \rightarrow \widetilde{M}_{f}$ are embeddings and their images are the two boundary edge paths of $\operatorname{star}(s)$ for a hyperplane $s$.

Definition 3.6. For each hyperplane $s_{n}$ there are lifts $\tilde{\phi}_{0}, \tilde{\phi}_{1}$ of $\phi_{0}, \phi_{1}$, respectively, such that $s^{-}=\tilde{\phi}_{0}(\mathbb{R}) \subset \operatorname{star}\left(s_{n}\right)$ and $s^{+}=\tilde{\phi}_{1}(\mathbb{R}) \subset \operatorname{star}\left(s_{n}\right)$. We will call $s^{-}$the smooth side and $s^{+}$the rugged side of the hyperplane $s_{n}$. 


\section{Edge Fibers}

In this section we discuss some properties of edge fibers that will play an important role in proving our main results.

First we remind the reader that we say that an edge fiber $\sigma$ corresponds to an edge $\tilde{E}_{j}$ if

- $\sigma \cap \tilde{E}_{j} \neq \emptyset$ and

- $\sigma \cap \tilde{E}_{m} \neq \emptyset$ for $\tilde{E}_{m} \in \tilde{G}$ implies that $m \geq j$.

It follows directly from the definition that if $\sigma$ is an edge fiber that corresponds to a copy of $\tilde{E}_{k}$, then $\sigma$ is an edge fiber for any $\tilde{E}_{k}$ such that $\tilde{E}_{k} \cap \sigma \neq \emptyset$, but not for any $\tilde{E}_{m}, m>k$ that $\sigma$ might cross. The edge fiber in Figure 2 corresponds to an edge $\tilde{E}_{i}$. Note also that edge fibers cannot intersect transversally. They are either disjoint or they coincide after a certain point.

Definition 4.1. An edge fiber $\sigma$ that corresponds to an edge $\tilde{E}_{j}$ is faithful if $\sigma \cap \tilde{E}_{m} \neq \emptyset$ for $\tilde{E}_{m} \in \tilde{G}$ implies that $m=j$.

REMARK 4.2. Note that we can define smooth and rugged sides of a faithful edge fiber in a similar fashion to our definition for hyperplanes.

In the remaining part of this section we investigate the properties of hyperplanes and dominating edge fibers in the mapping torus that are consequences of the bounded eigenray cancellation property of Kolchin maps, because those properties will play an important role in the proofs of our main results. First we will prove that if $\tilde{E}_{k}$ is a dominating edge for $\tilde{E}_{m}$ and if an edge fiber corresponding to a $\tilde{E}_{k}$ intersects a hyperplane $s_{m}$ corresponding to $\tilde{E}_{m}$, then $\tilde{E}_{k}$ is contained in an eigenray associated with a copy of $\tilde{E}_{m}$ at the level $r=\tilde{g}\left(\tilde{E}_{k}\right)$.

Lemma 4.3. Let $f: G \rightarrow G$ be a Kolchin map with the collection of topmost edges $\mathcal{E}=\left\{E_{i}, \ldots, E_{n}\right\}$ and let $E_{m} \in \mathcal{E}$. Let $E_{k}$ be the dominating edge for $E_{m}$. Assume that there is a dominating edge fiber $\sigma_{e}$ corresponding to an edge $e=\tilde{E}_{k}$ that intersects a hyperplane $s_{m}$ corresponding to an edge $\tilde{E}_{m}$ and such that $\tilde{g}(e) \geq \tilde{g}\left(s_{m} \cap \sigma_{e}\right)$ for a level map $\tilde{g}: \widetilde{M}_{f} \rightarrow \mathbb{R}$ (see Figure 4). Then $e$ is contained in the eigenray of the copy of $E_{m}$ that intersects $s_{m}$ and satisfies $\tilde{g}\left(\tilde{E}_{m}\right)=\tilde{g}(e)$.

Proof. First note that if an edge fiber corresponding to $\tilde{E}_{k}$ intersects an edge $\tilde{E}_{m}, m \geq k$ such that $\tilde{g}\left(\tilde{E}_{m}\right)=\tilde{g}\left(\tilde{E}_{k}\right)-1$, then both $\tilde{E}_{m}$ and $\tilde{E}_{k}$ are contained in a 2-cell $B_{m}$ with boundary label $\tilde{t}^{-1} \tilde{E}_{m} \tilde{t} \tilde{u}_{m}^{-1} \tilde{E}_{m}^{-1}$. Therefore $\tilde{E}_{k}$ is contained in $\tilde{f}\left(\tilde{E}_{m}\right)$ where $\tilde{g}\left(\tilde{E}_{m}\right)=\tilde{g}\left(\tilde{E}_{k}\right), \tilde{E}_{m}$ intersects $s_{m}$ and $\tilde{f}$ 


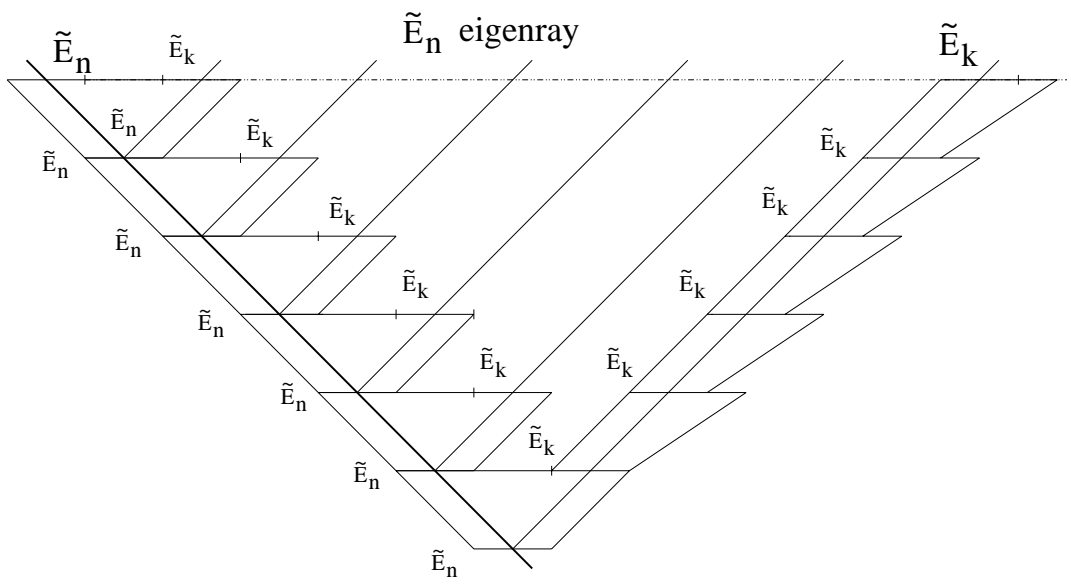

Figure 4: Edge fibers and eigenrays

is the lift of $f$ that fixes the initial endpoint of $\tilde{E}_{m}$. Moreover, the same reasoning implies that if an edge fiber corresponding to $\tilde{E}_{k}$ intersects a basic path $\tilde{E}_{m} \gamma \subset \tilde{G}$, height $(\gamma) \leq \operatorname{height}\left(u_{m}\right)$ and $\tilde{g}\left(\tilde{E}_{m} \gamma\right)=\tilde{g}\left(\tilde{E}_{k}\right)-1$, then $\tilde{E}_{k}$ is contained in $\tilde{f}\left(\tilde{E}_{m} \gamma\right)$, where $\tilde{f}$ is the lift of $f$ that fixes the initial endpoint of $\tilde{E}_{m}$. Applying induction we can conclude that $\tilde{E}_{k}$ is contained in $\tilde{f}^{i}\left(\tilde{E}_{m}\right)$ for $i=\tilde{g}\left(\tilde{E}_{k}\right)-\tilde{g}\left(\tilde{E}_{m}\right)$ and since $\tilde{E}_{k}$ is a topmost edge in $\tilde{u}_{m}$ then it is also contained in $\left[\tilde{f}^{i}(\gamma)\right]$ and therefore in the eigenray for $\tilde{E}_{m}$.

The following proposition is a consequence of the bounded eigenray cancellation property for Kolchin maps and Lemma 4.3.

Proposition 4.4. Let $f: G \rightarrow G$ be a Kolchin map growing polynomially with degree $d \geq 2$ with the collection of topmost edges $\mathcal{E}=\left\{E_{i}, \ldots, E_{n}\right\}$. There is a constant $L$, such that for any two hyperplanes $s_{i}$ and $s_{j}$ there are at most $L$ dominating edge fibers $\sigma$ that intersect both $s_{i}$ and $s_{j}$.

Proof. Let $s_{m}$ and $s_{i}$ be two distinct hyperplanes corresponding to the edges $\tilde{E_{m}}$ and $\tilde{E}_{i}$ from the collection of topmost edges and let $\mathcal{H}$ be a collection of all dominating edge fibers that intersect both $s_{m}$ and $s_{i}$. Let $P \in \sigma \cap\left(s_{m} \cup s_{i}\right), \sigma \in \mathcal{H}$, be a point such that $\tilde{g}(P)=\max \{\tilde{g}(T): T \in$ $\left.\sigma \cap\left(s_{m} \cup s_{i}\right), \sigma \in \mathcal{H}\right\}$ for a level function $\tilde{g}$. Without loss of generality we can assume that $P \in \sigma \cap s_{m}$. Let $Q \in \sigma \cap s_{m}$ such that $\tilde{g}(Q)=\min \{\tilde{g}(T)$ : $\left.T \in \sigma \cap s_{m}, \sigma \in \mathcal{H}\right\}$ and let $q=\tilde{g}(P)-\tilde{g}(Q)$ (Figure 5). Note that for every $r \in[\tilde{g}(Q), \tilde{g}(P)]$ there is a dominating edge fiber $\sigma$, corresponding to an edge $\tilde{E}_{k}$, that intersects $s_{m}$ at the level $r$. The intersection $s_{m} \cap \sigma$ is 


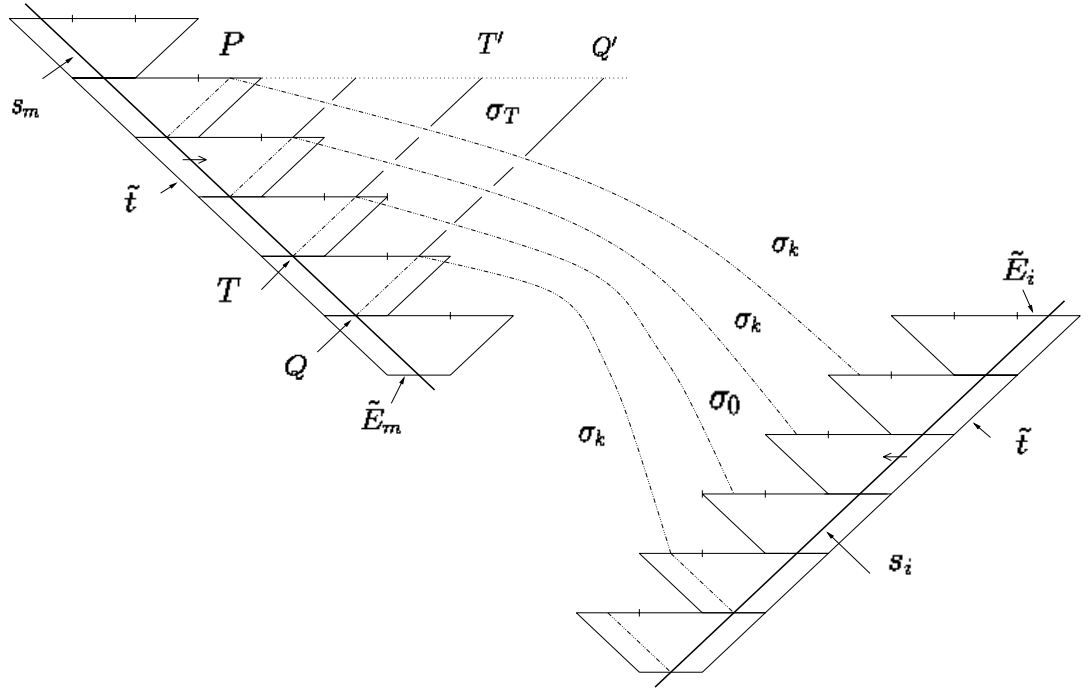

Figure 5: Bounded cancellation property in the case of two hyperplanes

contained in a 2-cell with boundary label $\tilde{t} \tilde{E}_{m} \tilde{t} \tilde{u}_{m}^{-1} \tilde{E}_{m}^{-1}$ and $\sigma$ intersects a copy of $\tilde{E}_{k}$ in the boundary of $B_{m}$. Let $T=\sigma_{0} \cap \tilde{E}_{k} \in B_{m}$. Let $\sigma_{T}$ be the faithful edge fiber corresponding to $\tilde{E}_{k}$ such that $T \in \sigma_{T}$ and let $T^{\prime}=$ $\sigma_{T} \cap \tilde{g}^{-1}\left(\tilde{g}(P)\right.$. Since there is an edge fiber corresponding to the edge $E_{k}$ that contains $T^{\prime}$ which intersects $s_{m}$, Lemma 4.3 implies that both $P$ and $T^{\prime}$ are in the eigenray corresponding to the copy of $\tilde{E}_{m}$ such that $\tilde{E}_{m} \cap s_{m} \neq \emptyset$ and $\tilde{g}\left(\tilde{E}_{m}\right)=\tilde{g}(P)=\tilde{g}\left(T^{\prime}\right)$. On the other side, by the same reasoning, we have also that both $P$ and $T^{\prime}$ are in the eigenray corresponding to the copy of $\tilde{E}_{i}$ such that $\tilde{E}_{i} \cap s_{i} \neq \emptyset$ and $\tilde{g}\left(\tilde{E}_{i}\right)=\tilde{g}(P)=\tilde{g}\left(T^{\prime}\right)$. Now the bounded cancellation property implies that $q<3$ or that $s_{i}=s_{m}$. Since we assumed that $s_{i} \neq s_{m}$ we conclude that $q<3$ and therefore there cannot be more than $3 M$ dominating edge fibers that intersect two different hyperplanes, where $M=\max \left\{\left|u_{j}\right|: k<j \leq n\right\}$.

REMARK 4.5. The statement of Proposition 4.4 is not true in the case $d=1$ in general, since infinitely many dominating edge fibers can intersect two faithful edge fibers corresponding to linearly growing edges. However, it follows from the bounded cancellation property and the proof of Proposition 4.4 that whenever there are more than $L$ dominating edge fibers $\sigma_{k}$ that intersect two different faithful edge fibers $\sigma_{i}$ and $\sigma_{j}$ corresponding to the edges $\tilde{E}_{i}$ and $\tilde{E}_{j}$, respectively, then $u_{i}=[\tau]^{p}, u_{j}=[\tau]^{q}$ for 
a Nielsen path $\tau$ and $p, q \in \mathbb{N}$.

The next lemma shows that if we have a finite collection of hyperplanes $\mathcal{H}$, containing $N$ hyperplanes, the number of dominating edge fibers that intersect two different hyperplanes in $\mathcal{H}$ is bounded by a linear function of $N$.

Lemma 4.6. Let $f: G \rightarrow G$ be a Kolchin map growing polynomially with degree $d \geq 2$ and $\left\{E_{i}, \ldots, E_{n}\right\}$ the collection of topmost edges. There is a constant $A \in \mathbb{R}$ such that if $\mathcal{H}$ is a collection of hyperplanes in $\widetilde{M}_{f}$ with $\operatorname{card}(\mathcal{H})=N$ then the number of dominating edge fibers that intersect two hyperplanes in the collection $\mathcal{H}$ is less than or equal to $A N$ (Figure 6 ).
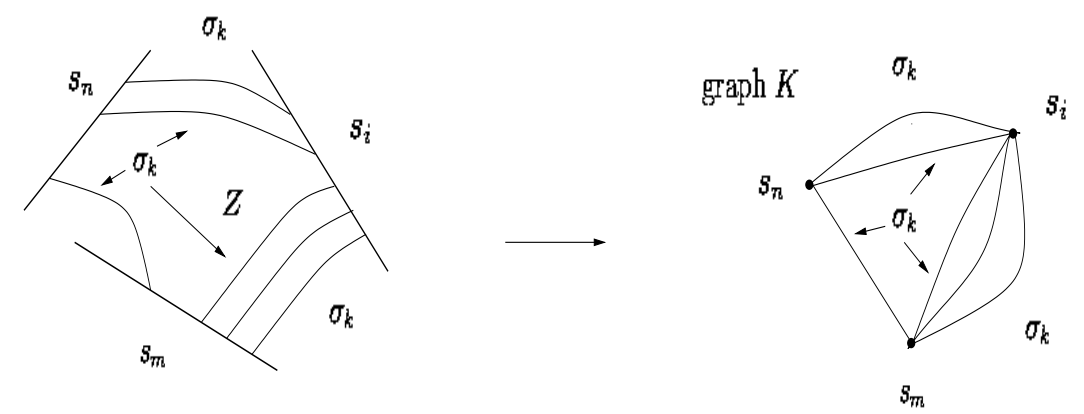

Figure 6: Bounded cancellation property in the case of $N$ hyperplanes

Proof. Since dominating edge fibers cannot intersect hyperplanes transversally, we can assume that $s \cap C l Z \neq \emptyset$ for any hyperplane $s \in \mathcal{H}$, where $Z$ is a vertex component $Z$ of $\widetilde{M}_{f}$. We can construct a graph $K$ (Figure 6 ) such that the vertices $V_{K}=\left\{v_{1}, \ldots, v_{k}\right\}$ of $K$ are hyperplanes in $\mathcal{H}$ and edges $E_{K}=\left\{e_{1}, \ldots, e_{p}\right\}$ correspond to dominating edge fibers that intersect hyperplanes in $\mathcal{H}$. The bounded cancellation property implies (Lemma 4.3) that there are at most $L$ edges in $K$ connecting two given vertices. Since the dominating edge fibers separate $Z$ and cannot cross each other, for every edge $e \in E_{K}$ connecting two vertices $v$ and $v^{\prime}$ there is a partition of $V_{K} \backslash\left\{v, v^{\prime}\right\}$ into two subsets $V_{1}, V_{2}$ such that no vertex in $V_{1}$ can be connected with a vertex in $V_{2}$. Moreover, it is not difficult to see that for a given vertex $v \in K$ there are at most two edges $e^{\prime}, e^{\prime \prime}$ with one endpoint at $v$ and the other at vertices $v^{\prime} \neq v^{\prime \prime}$ respectively with the property that one of the sets in the above partition is empty. This property follows from the 
fact that two edge fibers that intersect a hyperplane $s$ bound a segment of the hyperplane $s$. If there are three or more edge fibers intersecting $s$, one of them would have to separate the endpoints of the segment bounded by the other two, and therefore would also separate the corresponding edge fibers. It follows by induction, using the above properties, that there are at most $3(N-2) L$ edges in $K$.

We conclude this section with a lemma that will be used when discussing the case of linearly growing Kolchin maps.

Lemma 4.7. Let $\sigma_{i}$ and $\sigma_{j}$ be faithful edge fibers in $\widetilde{M}_{f}$ and $\tilde{g}$ a level function. Let $\gamma_{r}$ be a geodesic in $\tilde{G}$ connecting points $P_{r}=\sigma_{i} \cap \tilde{g}^{-1}(r)$ and $Q=\sigma_{j} \cap \tilde{g}^{-1}(r)$. Assume that all the dominating edge fibers that intersect $\gamma_{0}$ also intersect both $\sigma_{i}$ and $\sigma_{j}$ and that there are more than $L$ dominating edge fibers that intersect both $\sigma_{i}$ and $\sigma_{j}$. Then $\gamma_{r}$ is a concatenation of Nielsen paths for any $r \in \mathbb{R}$.

Proof. Let $\sigma_{i}$ and $\sigma_{j}$ be faithful edge fibers in $\widetilde{M}_{f}$ corresponding to edges $\tilde{E}_{i}$ and $\tilde{E}_{j}$. Remark 4.5 implies that if there are more than $L$ dominating edge fibers that intersect both $\sigma_{i}$ and $\sigma_{j}$, then $u_{i}=[\tau]^{p}, u_{j}=[\tau]^{q}$ for a Nielsen path $\tau$ and $p, q \in \mathbb{N}$. Let $E_{k}$ be the topmost edge in both $u_{i}$ and $u_{j}$. Then each of them splits into subpaths of the following type, where $\gamma$ is a subpath in $G_{k-1}: \gamma, E_{k} \gamma E_{k}^{-1}, E_{k} \gamma$, or $\gamma E_{k}^{-1}$. Note that the resulting subpaths are Nielsen and we can also consider paths that connect the midpoints of copies of $\tilde{E}_{k}$ in $u_{i}$ and $u_{j}$ to be Nielsen. But since all dominating edge fibers that intersect $\gamma_{0}$ also intersect $\sigma_{i}$ and $\sigma_{j}, \gamma_{0}$ is a concatenation of Nielsen paths. Since the endpoints of copies of $\tilde{E}_{i}$ and $\tilde{E}_{j}$ that intersect $\gamma_{0}$ are split points for their eigenrays, $\gamma_{r}$ is also a Nielsen path for any $r \in \mathbb{R}$.

\section{$5 \quad$ Nice Disks}

We want to decompose a disk $\Delta: D^{2} \rightarrow \widetilde{M}_{f}$ into subdisks contained in the mapping torus of a Kolchin graph with a smaller number of strata, and so we will cut the disk along the preimages in $D^{2}$ of hyperplanes in $\widetilde{M}_{f}$. Therefore the preimages of hyperplanes in $\widetilde{M}_{f}$ should be properly embedded arcs, which motivates the following terminology:

Definition 5.1. Let $f: G \rightarrow G$ be a Kolchin map. Let $\Delta: D^{2} \rightarrow \widetilde{M}_{f}$ be a disk with a boundary path $c$. The height of $\Delta$, height $(\Delta)$, is the height of a topmost edge $\tilde{E}_{k}$ in $\tilde{G}$ which satisfies $\Delta^{-1}\left(\tilde{E}_{k}\right) \neq \emptyset$. 
Definition 5.2. Let $f: G \rightarrow G$ be a Kolchin map and let $\Delta: D^{2} \rightarrow \widetilde{M}_{f}$ be a disk with boundary an edge path $c$ and height height $(\Delta)=n$. We will say that $\Delta$ is a nice disk if:

$1_{n}$ For any hyperplane $s_{n}, \Delta^{-1}\left(s_{n}\right)$ is a properly embedded arc in $D^{2}$.

$2_{n}$ The length of $\Delta\left(\Delta^{-1}\left(\cup\left\{s_{n}\right.\right.\right.$ a hyperplane $\left.\left.\}\right)\right)$ in $\widetilde{M}_{f}$ is minimal over all disks with boundary $c$ that satisfy $1_{n}$.

$1_{j}$ Let $Z=\cup\left\{\Delta^{-1}\left(\sigma_{i}\right): \sigma_{i}\right.$ an edge fiber corresponding to $\left.\tilde{E}_{i}, i>j\right\}$. $\Delta^{-1}\left(\sigma_{j}\right)$ consists of properly embedded arcs in components of $D^{2} \backslash Z$ for all faithful edge fibers corresponding to an edge $\tilde{E}_{j}$.

2 The length of $\Delta\left(\Delta^{-1}\left(\cup\left\{\sigma_{j}: \sigma_{j}\right.\right.\right.$ a faithful edge fiber for $\left.\left.\left.\tilde{E}_{j}\right\}\right)\right)$ in $\widetilde{M}_{f}$ is minimal over all disks with boundary $c$ that satisfy $1_{i}$ for all $i \geq j$ and $2_{i}$ for $i>j$.

REMARK 5.3. Let $f: G \rightarrow G$ be a Kolchin map with a collection of topmost edges $\left\{E_{i}, \ldots, E_{n}\right\}$. If $\Delta: D^{2} \rightarrow \widetilde{M}_{f}$ is a disk with boundary an edge path $c$ and height height $(\Delta)=1, i \leq 1 \leq n$, then a standard surgery argument implies that there is a nice disk $\Delta^{\prime}: D^{2} \rightarrow \widetilde{M}_{f}$ with boundary $c$ and height height $\left(\Delta^{\prime}\right) \leq 1$. The surgery is applied to remove simple closed curves in $\Delta^{-1}\left(s_{m}\right), i \leq m \leq n$.

REMARK 5.4. We can assume that if $\zeta$ is a preimage in a nice disk $\Delta: D^{2} \rightarrow$ $\widetilde{M}_{f}$ of a hyperplane $s_{i}$ then $\left.\Delta\right|_{\zeta}$ is injective, since otherwise an obvious surgery would give us a disk with a smaller total length of hyperplanes. A similar statement is true for edge fibers.

Lemma 5.5. Let $f: G \rightarrow G$ be a Kolchin map and let $\Delta: D^{2} \rightarrow \widetilde{M}_{f}$ be a nice disk with boundary an edge path $c$. Then the height of $\Delta$, height $(\Delta)$, is equal to the height of the boundary circuit $c$ as an edge path in $\tilde{G}$.

Proof. Since $\Delta$ is a nice disk, height $(\Delta)=n$ implies that $\tilde{E}_{n}$ is a topmost edge $\Delta$ intersects nontrivially, but it is also the topmost edge in $c$ because the preimages of $n$-hyperplanes are properly embedded arcs.

\section{Special Disks}

Let $f: G \rightarrow G$ be a Kolchin map that grows polynomially with degree $d \geq 2$. Let $\Delta: D^{2} \rightarrow \widetilde{M}_{f}$ be a nice disk with boundary $c$ and $h e i g h t(\Delta)=n$. The topmost edge decomposition of $M_{f}$ induces a decomposition of $D^{2}$ into subdisks contained in the preimages of various vertex components of $\widetilde{M}_{f}$. Since we want the resulting disks to have edge paths 
for boundaries, we will actually cut $D^{2}$ along the preimages of the edge paths parallel to the hyperplanes, that is, along the preimages of rugged and smooth sides of hyperplanes. As a result we will obtain subdisks (with edge paths as boundaries) in the preimages of various copies of the universal cover of the vertex space and subdisks contained in the preimage of $\operatorname{star}\left(s_{n}\right)$ for $s_{n}$ such that $\Delta^{-1}\left(s_{n}\right) \neq \emptyset$. We will refer to this decomposition as cutting along hyperplanes (Figure 7) and say that a subdisk $\Delta_{j}$ of $\Delta$, resulting from such a decomposition, is a special disk if its image is contained in a copy of a vertex space $\tilde{Y}_{v}$. Note that if $\Delta_{j}$ is a special disk result-

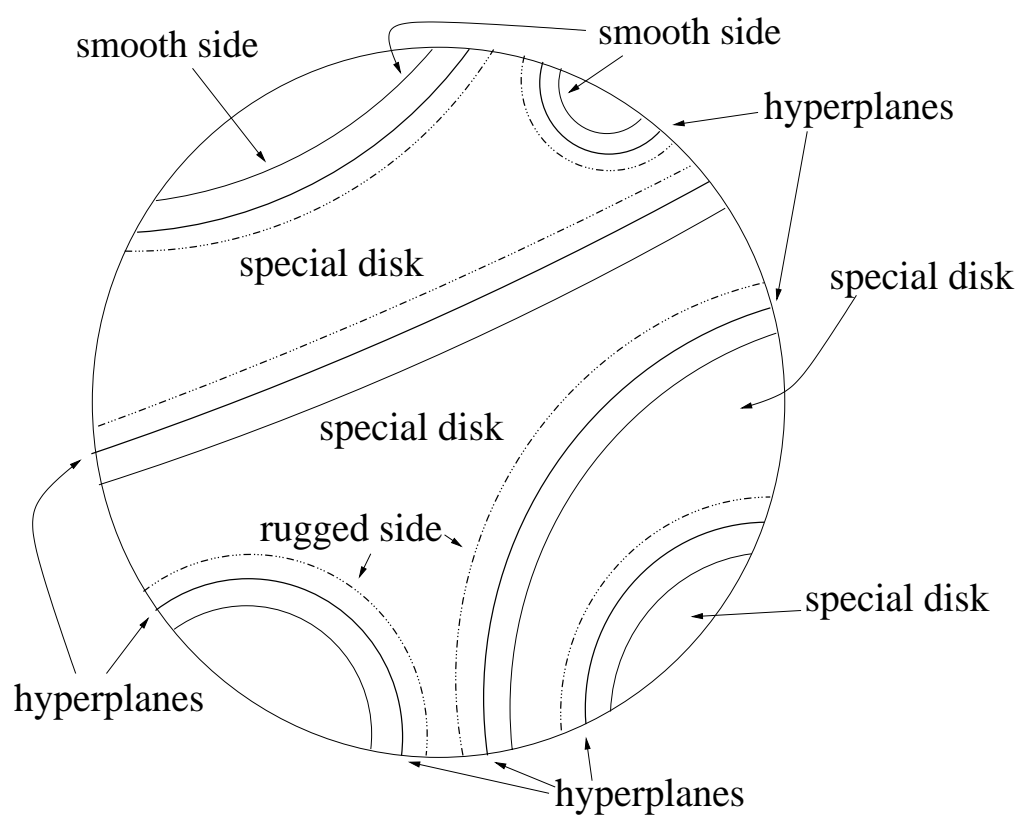

Figure 7: Cutting $\Delta$ along hyperplanes

ing from cutting $\Delta$ along the hyperplanes, then $\operatorname{Im}\left(\Delta_{j}\right)$ is contained in the mapping torus of a Kolchin map that grows polynomially with degree $d-1$. Let $\Delta$ be a nice disk and $\mathcal{Z}=\left\{\zeta_{1}, \ldots, \zeta_{p}\right\}$ the collection of all nonempty preimages of hyperplanes in $\widetilde{M}_{f}$. Say that the length of $\zeta_{i}, l_{i}=l\left(\zeta_{i}\right)$, of a preimage of a hyperplane $s^{i}$ is the number of 2-cells that $\Delta\left(\zeta_{i}\right)$ intersects. Note that the length of the corresponding segment of the smooth side of a hyperplane $s^{i}$ (as an edge path) is equal to $l\left(\zeta_{i}\right)$ and the length of the 
rugged side is less than equal to $\operatorname{Ml}\left(\zeta_{i}\right)$ where

$$
M=\max \left\{\left|u_{i}\right|: u_{i} \text { a suffix in } G\right\} .
$$

Also note that the area of a subdisk of $\Delta$ that is contained in $\operatorname{star}\left(s^{i}\right)$ is equal to the length of the preimage of the corresponding hyperplane $s^{i}$.

We will prove the following lemma:

Lemma 6.1. Let $f: G \rightarrow G$ be a Kolchin map that grows polynomially with degree $d \geq 2$. Let $\Delta: D^{2} \rightarrow \widetilde{M}_{f}$ be a nice disk with boundary $c$ and $\mathcal{Z}=\left\{\zeta_{1}, \ldots, \zeta_{p}\right\}$ the collection of all nonempty preimages of hyperplanes in $\widetilde{M}_{f}$. There is a constant $A \in \mathbb{R}$, which does not depend on $\Delta: D^{2} \rightarrow \widetilde{M}_{f}$, such that

$$
\sum_{i=1}^{i=p} l\left(\zeta_{i}\right) \leq A|c| .
$$

The above lemma will imply that for $\Delta_{j}, j=1, \ldots, J$, the collection of special disks resulting from cutting $\Delta$ along the hyperplanes,

$$
\sum_{j=1}^{J}\left|c_{j}\right| \leq K|c|,
$$

where $c_{j}$ is the boundary of $\Delta_{j}, j=1, \ldots, J$ and $K=2 M A+1$ a constant. Since the area in the rest of the components (those in the edge spaces) is linear, we may use induction on the height of a disk $\Delta$ in order to prove the quadratic isoperimetric inequality.

Proof of Lemma 6.1. Let $\Delta$ be a nice disk and $\mathcal{Z}=\left\{\zeta_{1}, \ldots, \zeta_{p}\right\}$ the collection of all nonempty preimages of hyperplanes in $\widetilde{M}_{f}$. Since the length of the preimage of a hyperplane is proportional to the number of preimages of dominating edge fibers that intersect it, we will follow the preimages of the edge fibers corresponding to a dominating edge $\tilde{E}_{k}$ that intersect some $\zeta_{i} \in \mathcal{Z}$. Note that in a nice disk any preimage of a dominating edge fiber that intersects $\zeta_{i} \in \mathcal{Z}$ must also intersect the boundary of $D^{2}$ or another $\zeta_{m} \in \mathcal{Z}$. Since only one dominating edge fiber can intersect the corresponding edge in the boundary, we will need a bound on the number of edge fibers that intersect two different preimages of hyperplanes and not the boundary of $D^{2}$. Lemma 4.6 implies that there is a constant $A \in \mathbb{R}$ such that at most $A p$ edge fibers corresponding to dominating edges can intersect two different hyperplanes whose preimages are in the collection $\mathcal{Z}$. We will show that if $\zeta_{1}, \zeta_{2} \in \mathcal{Z}$ are preimages of hyperplanes $s^{1}, s^{2} \subset \widetilde{M}_{f}$ and there is a preimage $\xi$ of a dominating edge fiber that intersects both $\zeta_{1}$ and $\zeta_{2}$, then it follows from the properties of nice disks that $s^{1} \neq s^{2}$. 
That is, if $s^{1}=s^{2}$, then it would follow from the properties of dominating edge fibers (specifically, that they can intersect a particular hyperplane only once) that $\Delta(\xi) \cap s^{1}=\Delta(\xi) \cap s^{2}$, and we would be able to do a surgery (see Figure 8) removing a tubular neighborhood $N$ of $\xi$ and obtain a disk with smaller total length of the preimages of edge fibers, which is a contradiction to our assumption that $\Delta$ is a nice disk.

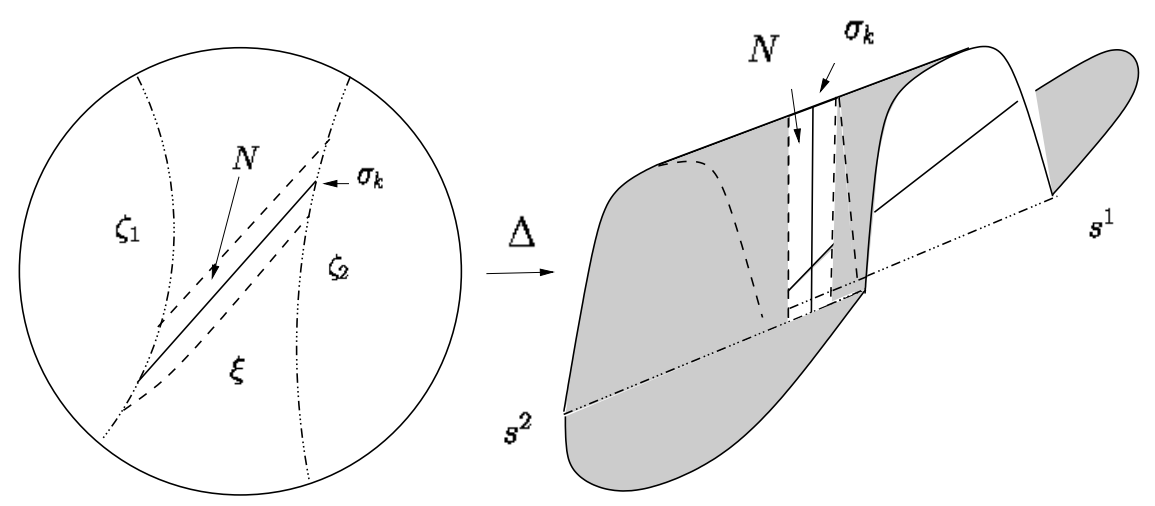

Figure 8: The surgery that reduces the length of the preimages in $D^{2}$ of edge fibers

Proposition 6.2. Let $f: G \rightarrow G$ be a Kolchin map that grows polynomially with degree $d \geq 2$. Let $\Delta: D^{2} \rightarrow \widetilde{M}_{f}$ be a nice disk with boundary $c$. There is a constant $K \in \mathbb{R}$, which does not depend on $\Delta: D^{2} \rightarrow \widetilde{M}_{f}$, such that if $\Delta_{j}, j=1, \ldots, J$, is the collection of special disks resulting from cutting $\Delta$ along the hyperplanes, with respective boundaries $c_{j}$, then

$$
\sum_{j=1}^{J}\left|c_{j}\right| \leq K|c|
$$

Proof. The claim of the proposition follows directly from Lemma 6.1 since

$$
\sum_{j=1}^{J}\left|c_{j}\right| \leq \sum_{i=1}^{i=p} l\left(\zeta_{i}\right)+M \sum_{i=1}^{i=p} l\left(\zeta_{i}\right)+|c|
$$

where $\mathcal{Z}=\left\{\zeta_{1}, \ldots, \zeta_{p}\right\}$ is the collection of all nonempty preimages of hyperplanes in $\widetilde{M}_{f}$. 


\section{Linearly Growing Kolchin Maps}

In order to complete the proof of the quadratic isoperimetric inequality for the mapping tori of Kolchin maps we must prove that the mapping torus of a linearly growing Kolchin map $f: G \rightarrow G$ satisfies the quadratic isoperimetric inequality.

Proposition 7.1. Let $f: G \rightarrow G$ be a linearly growing Kolchin map and $M_{f}$ the mapping torus of $f$. Then $M_{f}$ satisfies the quadratic isoperimetric inequality.

Proof. We will use the approach from $[\mathrm{BF}]$ based on the standard decomposition of $M_{f}=X$ as a graph of spaces. (The edge space is $X_{e}=G$, and the vertex space $X_{v}$ is obtained from the mapping cylinder $C_{f}=G \times I /(x, 1) \sim f(x)$ by attaching a collar to the (top) $G$.) Let $c$ be an edge path. According to $[\mathrm{BF}, \mathrm{p} .90]$ there is a disk $\Delta: D^{2} \rightarrow \tilde{X}$ with boundary $c$ that is transverse to $\cup\left\{\tilde{X}_{e} \mid e \in \operatorname{Edge}(T)\right\}$ and thus divides $D^{2}$ into regions which map into the negatively curved $\tilde{X}_{v}$ 's. Set $\mathcal{W}=\Delta^{-1}\left(\cup\left\{\tilde{X}_{e} \mid e \in \operatorname{Edge}(T)\right\}\right)$. In this section we will assume that a nice disk $\Delta$ also has the property that the set $\mathcal{W}$ consists of properly embedded arcs in $D^{2}$, and we will call each component of $\mathcal{W}$ a wall in $D^{2}$ (Figure 9 ).

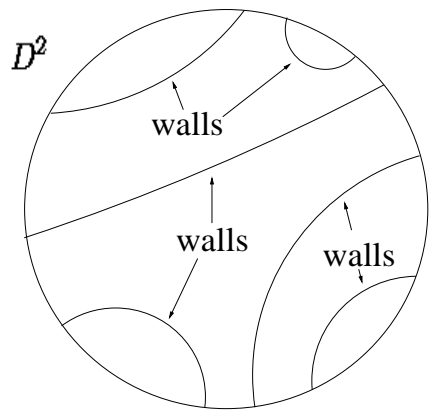

Figure 9: Walls in $D^{2}$

Let $\Delta: D^{2} \rightarrow \widetilde{M}_{f}$ be a nice disk with boundary an edge path $c$. Note that since every component of $\Delta\left(D^{2} \backslash(\cup \mathcal{W})\right)$ is contained in a negatively curved space, the closures of the components of $\Delta\left(D^{2} \backslash(\cup \mathcal{W})\right)$ have areas bounded by a constant times the length of their boundaries. Therefore we will first prove that there is a constant $K \in \mathbb{R}$ such that the length of any wall is bounded by $K|c|$. Since there are at most $|c|$ walls in $D^{2}$, this will imply that the area of $D^{2}$ is bounded by $K|c|^{2}$. 
To complete the proof we will need the following two lemmas.

Lemma 7.2. Let $f: G \rightarrow G$ be a linearly growing Kolchin map, $M_{f}$ the mapping torus of $f$ and let

$$
M=\max \left\{\left|u_{i}\right|: u_{i} \text { a suffix in } G\right\} .
$$

Let $\Delta: D^{2} \rightarrow \widetilde{M}_{f}$ be a nice disk with boundary an edge path $c$ and $w \subset \mathcal{W}$ a wall in $D^{2}$. Then there is a subdisk $D^{2 \prime}$ of $D^{2}$ such that

- $w \subset D^{2^{\prime}}$.

- All preimages of faithful edge fibers in $D^{2 \prime}$ intersect $w$.

- $\left|c^{\prime}\right| \leq M|c|$ where $c^{\prime}$ is the boundary circuit of $\Delta^{\prime}$.

Proof. Assume that a preimage of a faithful edge fiber $\sigma_{i}$ is contained in $D^{2}$ and that it does not intersect $w$ (see Figure 10). We know that $\Delta^{-1}\left(\sigma_{i}\right)$

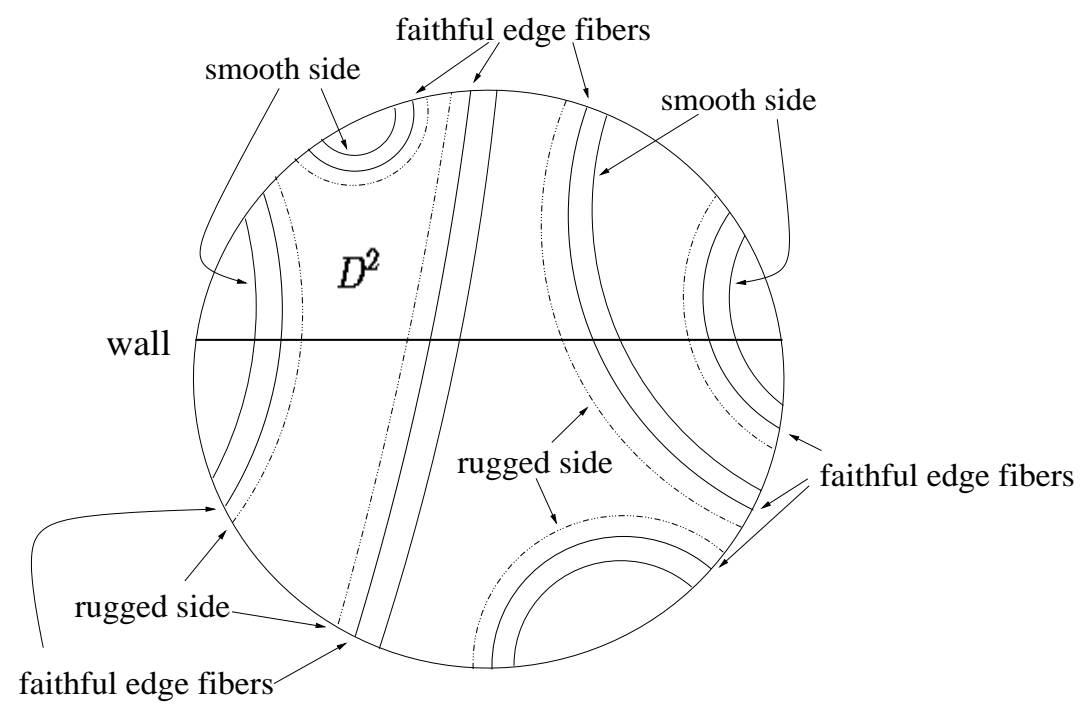

Figure 10: Cutting $D^{2}$ along preimages of faithful edge fibers

is a properly embedded arc, so it separates $D^{2}$ into two components and exactly one of them, say $D_{w}$, contains $w$. But then $D_{w}$ also contains the preimage of a path $\omega_{1}=\tilde{t}^{l_{i}}$ or $\omega_{2}=\left(\tilde{t} \tilde{u}_{i}^{-1}\right)^{l_{i}}$. We can cut $D^{2}$ along the preimage of $\omega_{1}$ or $\omega_{2}$, whichever is contained in $D_{w}$.

Lemma 7.3. Let $f: G \rightarrow G$ be a linearly growing Kolchin map and $M_{f}$ the mapping torus of $f$. There is a constant $K \in \mathbb{R}$ such that if $\Delta: D^{2} \rightarrow \widetilde{M}_{f}$ is a nice disk with boundary an edge path $c$ then the length of any wall is bounded by $K|c|$. 
Proof. First note that since all the edges are linearly growing there is a constant $B$ such that $\left|f^{k}\left(E_{m}\right)\right| \leq B k$ for all edges $E_{m} \in G$ and any $k \in \mathbb{Z}$. Let $\Delta: D^{2} \rightarrow \widetilde{M}_{f}$ be a nice disk with boundary an edge path $c$ and let $w$ be a wall in $D^{2}$.

Let $\mathcal{F}=\left\{\xi_{1}, \ldots, \xi_{p}\right\}$ be the collection of all nonempty preimages in $D^{2}$ of faithful edge fibers. Lemma 7.3 implies that we can assume that every $\xi \in \mathcal{F}$ intersects $w$. Let $F=\cup\{\xi: \xi \in \mathcal{F}\}$, let $D_{0}$ be a component of $D^{2} \backslash F$, and let $v_{0}=D_{0} \cap w$. We claim that $\left|v_{0}\right| \leq K\left|c \cap D_{0}\right|$ for a constant $K$ which does not depend on $\Delta$. Let $\xi_{i}$ and $\xi_{j}$ be two preimages of faithful edge fibers, corresponding to edges $\tilde{E}_{i}$ and $\tilde{E}_{j}$, respectively, that bound the component $D_{0}$ (see Figure 11).

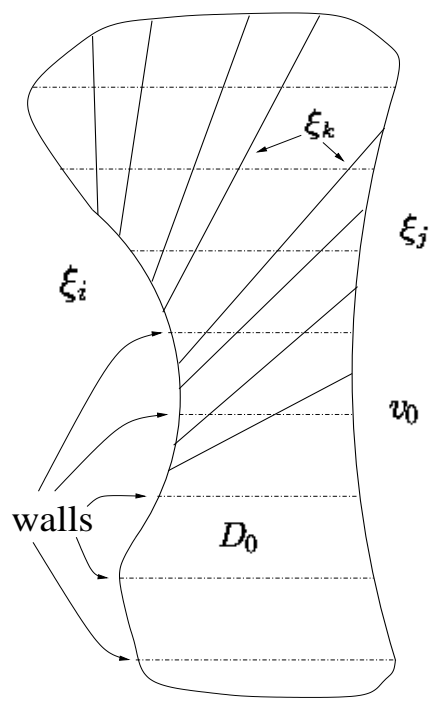

Figure 11: Walls and edge fibers in the linear case

We will discuss two different cases, first the case when the number of preimages of dominating edge fibers for $\tilde{E}_{i}$ that intersect $\xi_{j}$ is less than or equal to the constant $L$ from Proposition 4.4, and secondly the case when it is greater than $L$.

Case 1: Assume that the number of preimages of dominating edge fibers for $\tilde{E}_{i}$ that intersect $\xi_{j}$ is less than or equal to the constant $L$ from Proposition 4.4. In this case, it follows in the same fashion as in Lemma 6.1 that the length of each of $\xi_{i}$ and $\xi_{j}$ is less than or equal to $\left|c \cap D_{0}\right|+L$. Therefore the total number of walls that $D_{0}$ intersects is less than equal 
to $3\left|c \cap D_{0}\right|+2 L$. Since $f: G \rightarrow G$ is a linearly growing Kolchin map, $\left|v_{0}\right| \leq 2 B N_{0}$, where $N_{0}$ is the total number of walls in $c \cap D_{0}$, which implies our claim in this case.

Case 2: Assume now that the number of preimages of dominating edge fibers for $\tilde{E}_{i}$ that intersect $\xi_{j}$ is greater than $L$. In this case the bounded cancellation property for Kolchin maps (see Proposition 4.4) implies that $u_{i}=[\tau]^{p}, u_{m}=[\tau]^{q}$ for a Nielsen path $\tau$ and $p, q \in \mathbb{N}$. Let $E_{k}$ be the topmost edge in $u_{i}$, which is the same as the topmost edge in $u_{j}$. Let $b_{i}$ be the number of occurrences of $\tilde{E}_{k}$ in $\tilde{u}_{i}$ and $b_{j}$ be the number of occurrences of $\tilde{E}_{k}$ in $\tilde{u}_{j}$. Without loss of generality we can assume that $b_{i} \geq b_{j}$. Let $\tilde{g}$ be a level map such that $\tilde{g}(\Delta(w))=0$ and assume also that if $P \in D^{2}$ is a point such that $P=\xi_{i} \cap \xi_{k}, \xi_{k}$ being a preimage in $D_{0}$ of a dominating edge fiber $\sigma_{k}$ that intersects $v_{0}$, then $\tilde{g}(\Delta(P))<0$. This implies that for any preimage of a dominating edge fiber $\xi_{k}$ that intersects $v_{0}$ as well as the rugged side of $\xi_{j}$ at a point $Q=\xi_{j} \cap \xi_{k}$, we have that $\tilde{g}(\Delta(Q)) \geq 0$.

We will show that $\left|v_{0}\right| \leq M n_{v}$ where $n_{v}$ is the number of preimages of dominating edge fibers that intersect $v_{0}$. First note that the length of $v_{0}$ is less than or equal to the number of preimages of edge fibers that it intersects. Let $n_{i}$ be the number of preimages of dominating edge fibers that intersect both $\xi_{i}$ and $v_{0}$. Since $f$ is a linearly growing Kolchin map, the total number of edge fibers that intersect both $v_{0}$ and $\xi_{i}$ is less than or equal to $M n_{i}$. Let $n_{j}$ be the number of preimages of dominating edge fibers $\sigma_{k}$ that intersect $v_{0}$ and do not intersect $\xi_{i}$. Note that, since there are no faithful edge fibers in $D_{0}$, all preimages of edge fibers that do not intersect $\xi_{i}$ must intersect $\xi_{j}$. This implies that the number of edge fibers that intersect $v_{0}$ and not $\xi_{i}$ is less than or equal to $M n_{j}$. Therefore we need to show that $n_{j} \leq\left|c \cap D_{0}\right|$ and $n_{i} \leq\left|c \cap D_{0}\right|$. Note that the first inequality follows directly from the definition of $n_{j}$, since all such preimages of dominating edge fibers must intersect the boundary. We still need to show that $n_{i} \leq\left|c \cap D_{0}\right|$. Let $\tilde{g}\left(\xi_{i}\right)=\left[r_{1}, r_{2}\right]$. Since we assumed that $b_{i} \geq b_{j}$, there are more dominating edge fibers in $D_{0}$ that intersect $\xi_{i}$ than those that intersect $\xi_{j}$ at each level $r \in\left[0, r_{2}\right]$. Therefore there are more than $n_{i}$ dominating edge fibers that intersect the boundary or $\xi_{j}$ at a level $r \geq r_{2}$. The number of edge fibers that intersect $\xi_{j}$ at a level $r \geq r_{2}$ is bounded by a constant times the number of walls that intersect only $\xi_{j}$. Since walls are properly embedded arcs, any wall that intersects $\xi_{j}$ and not $\xi_{i}$ has to intersect an edge labeled $\tilde{t}$ in $c \cap D_{0}$. Therefore, the number of walls that intersect only $\xi_{j}$ is bounded by the number of edges labeled $\tilde{t}$ in $c \cap D_{0}$ and we can again conclude that 
$\left|v_{0}\right| \leq M\left|c \cap D_{0}\right|$, which completes the proof of the lemma.

Now we can complete the proof of Proposition 7.1. Since Lemma 7.3 implies that the length of any wall is bounded by $K|c|$, the area of the disk is bounded by

$$
\operatorname{Area}(\Delta) \leq N K(|c|),
$$

where $N$ is the total number of walls in the disk. Therefore

$$
\operatorname{Area}(\Delta) \leq K|c|^{2}
$$

which proves our claim.

We conclude by the proof of our main theorem:

Theorem 7.4. Let $f: G \rightarrow G$ be a Kolchin map that grows polynomially with degree $d$. Then the mapping torus $M_{f}=G \times I /{ }_{(x, 0) \sim(f(x), 1)}$ satisfies the quadratic isoperimetric inequality.

Proof. Let $f: G \rightarrow G$ be a Kolchin map that grows polynomially with degree $d$ and let $\Delta: D^{2} \rightarrow \widetilde{M}_{f}$ be a disk with boundary an edge path $c$. Then there is a nice disk $\Delta^{\prime}: D^{2} \rightarrow \widetilde{M}_{f}$ with boundary $c$ and of height height $\left(\Delta^{\prime}\right) \leq$ height $(\Delta)$. If $f$ is a linearly growing Kolchin map, Proposition 7.1 implies the claim of the theorem. If $d \geq 2$, Proposition 6.2 implies that we can decompose $\Delta^{\prime}$ and get a collection $\Delta_{j}, j=1, \ldots, J$, of special discs with boundaries $c_{j}$ such that $\Delta_{j}$ is contained in the mapping torus of a Kolchin map growing polynomially with degree less than or equal to $d-1$ for any $j=1, \ldots, J$ and

$$
\sum_{j=1}^{J}\left|c_{j}\right| \leq K|c|
$$

for a constant $K \in \mathbb{R}$, which does not depend on $\Delta: D^{2} \rightarrow \widetilde{M}_{f}$. Now, by induction we have that

$$
\operatorname{Area}\left(\Delta^{\prime}\right) \leq \sum_{j=1}^{J} \operatorname{Area}\left(\Delta_{j}\right) \leq A\left|c_{j}\right|^{2} \leq B|c|^{2}
$$

for constants $A, B \in \mathbb{R}$.

Acknowledgment I would like to thank Mladen Bestvina for suggesting the problem and for many helpful and stimulating discussions.

\section{References}

[BF] M. Bestvina, M. Feighn, A combination theorem for negatively curved groups, J. Differential Geometry 35 (1992), 85-101. 
[BFH1] M. Bestvina, M. Feighn, M. Handel, The Tits alternative for $\operatorname{Out}\left(F_{n}\right)$ I: Dynamics of exponentially growing automorphisms, Annals of Math. 151 (2000), 517-623.

[BFH2] M. Bestvina, M. Feighn, M. Handel, The Tits alternative for $\operatorname{Out}\left(F_{n}\right)$ II: A Kolchin theorem, preprint (1996).

[BH] M. Bestvina, M. Handel, Train tracks and automorphisms of free groups, Ann. Math. 135 (1992), 1-51.

[Br] M.R. BRIDson, Optimal isoperimetric inequalities for abelian-by-free groups, Topology 34 (1995), 547-564.

[BrG] M.R. Bridson, S.M. Gersten, The optimal isoperimetric inequality for torus bundles over the circle, Quart. J. Math. Oxford Ser.(2) 47 (1996), $1-23$.

[ECHLPH] D.B.A. Epstein, J.W. Cannon, D.F. Holt, S.V.F. Levy, M.S. Paterson, W.P. Thurston, Word Processing in Groups, Jones and Bartlett Publishers, 1992.

Nataša Macura, Department of Mathematics, The University of Michigan, East Hall, 525 East University Ave., Ann Arbor, MI 48109-1109, USA

Submitted: February 1999 Final version: September 1999 